\title{
LA INDICACIÓN FORMAL COMO PUNTO DE PARTIDA DE LA INVESTIGACIÓN FENOMENOLOGÍA ${ }^{1}$
}

\author{
Francisco Abalo C. ${ }^{2}$
}

\begin{abstract}
RESUMEN: El presente artículo se centra en algunos de los aspectos centrales de la concepción heideggeriana de la indicación formal. Como es sabido, el filósofo toma como punto de partida en una de las más tempranas exposiciones de este metaconcepto, una explicación delimitativa frente a otras operaciones conceptuales (generalización y formalización). Se revisará críticamente la explicación que Heidegger hace de la generalización a diferencia de la formalización, destacando especialmente que no se trata aquí de una mera distinción entre generalización y formalización, sino de una estructura de fundamentación. En segundo lugar se argumenta que la crítica de Heidegger no se dirige directamente contra la formalización, sino contra el contexto metódico al cual sirve. Por último, a modo de conclusión, se intenta mostrar que para Heidegger concebir la indicación formal como principio (metódico) de la investigación fenomenológica, supone que ella es, por vía negativa, una radicalización de la tendencia a lo formal.
\end{abstract}

PALABRAS CLAVE: Heidegger. Husserl. Fenomenología. Hermenéutica. Indicación formal.

\section{INTRODUCCIÓN}

El estudio metódico-sistemático de las Frühe freiburger Vorlesungen ${ }^{3}$, desarrollado ya por varios estudiosos, ${ }^{4}$ ha puesto en primer plano determinados aspectos y conexiones del pensamiento de Heidegger que permite dimensionar de forma más certera el alcance y los problemas inmanentes de este proyecto filosófico. Así, por ejemplo, la evidente relevancia de la fenomenología husserliana sobre el proyecto hiedeggeriano ha podido ser determinada

${ }^{1}$ El siguiente artículo ha sido elaborado en el marco del proyecto "Apoyo a la sistematización de la investigación” financiado por PROA /2015, VID-Universidad de Chile

2 Profesor Asistente/Universidad de Chile. Facultad de Filosofía y Humanidades, Departamento de Filosofía. Algunas publicaciones: Posible vía de solución al problema epistemológico de la Filosofía de la Historia hegeliana. Revista de Filosofia Aurora, p. 425-434, 2015; Relevancia y estatuto de lo conceptual en la Filosofía de la Historia de Hegel, Mutatis Mutandis: Revista Internacional de Filosofía, p. 59-76, 2014. E-mail: fjabalocea@gmail.com

3 Las Frühe freiburger Vorlesungen (Lecciones tempranas de Friburgo; en adelante FFV) corresponden a los volúmenes que van desde 56/57 al 63 de la Gesamtausgabe (GA) editada por Klostermann. Citaremos según la forma estándar en los estudios heideggerianos. Lo mismo vale para las citas de Husserl. Todas las traducciones corresponden al autor de este artículo, salvo que se indique expresamente el nombre de traductor correspondiente.

4 En relación a las líneas básicas de este enfoque dentro de los estudios heideggerianos, cf. (RODRÍGUEZ 1997, p. 14-15; DE LARA 2008, p. 15-20).

http://dx.doi.org/10.1590/S0101-31732017000400005 
de una forma mucho más específica, de tal manera que precisamente a la luz de estas lecciones es posible afirmar que, más que constituir una mera influencia, "[...] la fenomenología de Husserl es la filosofía de referencia en el proyecto de la hermenéutica de la facticidad.” (RODRÍGUEZ, 1997, p.15). Esta tesis, a mi juicio correcta, implica más que una constatación genéticoevolutiva, por lo demás bastante obvia; pues considera el proyecto filosófico de Heidegger, por lo menos a la altura de estas lecciones iniciales, como un esfuerzo de transformación de la fenomenología, lo cual supone a su vez un enfrentamiento crítico con los principios mismos desde los cuales es posible proyectar este tipo de investigación. Es mérito precisamente de este enfoque sistemático el haber puesto en el centro de la discusión la peculiar motivación de las FFV por apropiarse y garantizar metódicamente los propios principios que la hacen posible como investigación fenomenológica.

Uno de los ejes principales del pensamiento de Heidegger a estas alturas es su tratamiento expreso del estatuto y función de los conceptos filosóficos. En efecto, mediante el término "indicación formal" (formale Anzeige) Heidegger acuña su concepción de lo que constituye, en el decir de R. Rodríguez, un metaconcepto (RODRÍGUEZ, 2015, p. 59) que como tal hace expreso el "[...] sentido fundamental [...] de todos los conceptos filosóficos y tramas conceptuales.” (GA 9, p. 11). Por una parte, la indicación formal podría quedar enmarcada de forma específica en el "[...] difícil problema (nunca antes tratado) de la formación fenomenológica de conceptos” (GA 58, p. 219) y constituir, siguiendo en esto la lúcida indicación de Gethmann, la clave para comprender la "[...] teoría semántica específica de los conceptos filosóficos" en Heidegger (GETHMANN, 1993, p. 261). Por otra, sin embargo, este difícil problema cobra su verdadero sentido ahí donde se lo ve a partir de un contexto más amplio. Para Heidegger, como lo han hecho notar varios estudios, el problema de los conceptos filosóficos es indisoluble del problema básico de cómo la investigación fenomenológica accede y se mantiene fiel a su propio campo de problemas. ${ }^{5}$ En efecto, la indicación formal pertenece a la "[...] teoría del método fenomenológico mismo” (GA 60, p. 55), y esto en la medida en que precisamente para Heidegger el problema "[...] más candente y nunca cancelable, más originario y fundamental de la fenomenología es ella misma para sí misma." (GA 58, p. 1/traducción F. de Lara). Por tanto, las consideraciones metaconceptuales que Heidegger hace bajo el nombre de "indicación formal"

5 Para esto cf. (RODRÍGUEZ, 1997, p.19 ss.; DE LARA, 2008, p. 86 ss.; XOLOCOTZI, 2004, p. 107 ss; en especial GETHMANN, 1993, p. 267 ss). 
no responden sólo a la pregunta genérica de qué sean los conceptos filosóficos y cuál sea en general su génesis, sino que su importancia radica en que arrojan luz sobre el método fenomenológico mismo en la medida en que hacen explícita la función que los conceptos filosóficos tienen y que, de hecho, están teniendo al interior de una investigación fenomenológica en concreto. En este sentido, la indicación formal es ella misma "[...] un momento metódico de la explicación fenomenológica en cuanto tal.” (GA 60, p. 63).

En el siguiente artículo me centraré especialmente en este último aspecto, a saber, el que pone de relieve a la indicación formal como momento metódico de la investigación, para mostrar que, al menos en parte, el proyecto radicalizador de la fenomenología heideggeriana depende de enfrentar el problema de los conceptos filosóficos desde el punto de vista de la problemática de los principios metódicos de investigación filosófica. En principio, es desde esta perspectiva que se puede entender la caracterización inicial de la indicación formal como "[...] punto de partida de la explicación fenomenológica" [Ansetzen des phänomenologische Explikation; destacado por Heidegger], 9GA 60, p.64). En orden a esto, intentaré reconstruir las líneas argumentales principales que están contenidas en la que sería la primera presentación expresa y sistemática de este metaconcepto; me refiero en concreto a la exposición contendida en Phänomenologie des religiösen Leben (GA60, p. 55-5). Como es sabido, Heidegger toma como punto de arranque para su aclaración la distinción husserliana entre "generalización" y "formalización", lo cual orienta el tratamiento en la dirección de una delimitación negativa, caracterizando de esta forma la propia indicación formal por contraste con dichas operaciones conceptuales. A mi juicio, esta estrategia de presentación tiene dos consecuencias decisivas: por una parte, si bien la distinción husserliana marca para Heidegger una dirección para una posible radicalización metódica de la fenomenología, esto mismo implica una crítica fundamental a un determinado aspecto metódico clave para la pretensión científica de la fenomenología de Husserl; por otra parte, a partir del sentido de esta explicación delimitativa queda realzado sobre todo el carácter "formal" de la indicación formal—que algunos autores han llamado, el carácter "disuasivo"-lo cual se conecta de manera muy estrecha con el problema de los conceptos filosóficos como principios (metódicos).

De esta manera, el trabajo que presento a continuación contiene tres partes: 1) intentaré presentar críticamente la exposición que Heidegger hace de

\footnotetext{
${ }^{6}$ La más completa reconstrucción sistemática de todas las funciones implicadas en la indicación formal se encuentra en De Lara (2008, p.177-199).
} 
la generalización, para mostrar algunos aspectos problemáticos, especialmente destacando que no se trata aquí de una mera distinción entre generalización y formalización, sino de una estructura de fundación en la que, en cierto sentido, la primera supone a la última; 2) a partir de ello, intentaré mostrar que la crítica de Heidegger no se dirige directamente contra la formalización, sino contra el contexto metódico al cual sirve, contexto que inadvertidamente subvierte la estructura de fundación de estas dos operaciones conceptuales; 3) por último, y a modo de conclusión, intentaré mostrar de forma muy escueta que el hecho de que la indicación formal sea, por vía negativa, una radicalización de la tendencia a lo formal, implica para Heidegger concebirla como el principio (metódico) de la investigación fenomenológica.

\section{Discusión CRítica de la “GENERALIZaCión”}

El problema que tiene a la vista Heidegger la primera vez que introduce sistemáticamente la noción de indicación formal es el siguiente: ¿Cómo se relaciona lo histórico con la vida fáctica? (GA 60, p. 54). Pero este problema que se ofrece en el primer plano, implica un problema en un segundo plano más fundamental, es decir, implica un problema tal que recién desde la dilucidación de este problema es posible la resolución del primero. En efecto, en esta pregunta parece como si lo histórico fuese mentado en la forma de algo "universal" (allgemein), es decir, como ese conjunto unitario de determinaciones-por ejemplo, "ser algo que deviene en el tiempo"con las que califica cualquier cosa que pertenezca a esta región dentro de la totalidad de lo ente. Tradicionalmente, desde la perspectiva de Heidegger, esta es la figura predominante en la que se reconocen los conceptos. ${ }^{7}$ El filósofo, poniendo en cuestión el carácter universal del concepto de "histórico", avanza con su cuestionamiento hacia el supuesto carácter universal de todo concepto filosófico: "Es problemático [...] esta forma de universalidad es filosóficamente algo de principio.” (GA 60, p. 56). Parafraseando esto último, la pregunta concierne a la posibilidad de que la filosofía, desde el punto de

\footnotetext{
${ }^{7}$ Como es evidente, sin que haya necesariamente una distinción expresa en Heidegger al respecto, el problema metaconceptual de las formas de los conceptos arrastra consigo el de su forma de articulación lingüística: la definición. Es en la figura del problema del sentido y estructura de la definición filosófica que el autor aborda el problema en (GA 61, p.13-40) bajo la rúbrica de "la tarea de la definición". Es, asimismo, bien conocida la presencia de la objeción heideggeriana a la estructura tradicional de la definición (genero-especie) al inicio de Ser y tiempo (HEIDEGGER, 1993, p. 3)
} 
vista de su principio, opere exclusivamente o en absoluto con universales. ${ }^{8}$ Lo filosóficamente relevante en esta actitud escéptica inicial apunta, pues, a la aparente univocidad en la forma misma de los conceptos, y con ello abre al menos como posible que se conciban filosóficamente otras modulaciones metaconceptuales. Esto de hecho no es para Heidegger una mera posibilidad esbozada en el aire, sino que cuenta con distinciones bien acreditadas en el campo fenoménico descubierto por la fenomenología de Husserl.

Así pues, la condición de posibilidad de esta pregunta está ya de forma embrionaria en una diferencia que recoge Heidegger de Husserl, al cual cita expresamente en este punto. ${ }^{9}$ Se trata de la diferencia entre "generalización" (Generalisierung) y "formalización" (Formalisierung). En este sentido, la indicación formal intenta avanzar en la dirección de una mayor originalidad, dirección que precisamente se abre a partir de la diferencia entre generalización y formalización. Esto implica, por tanto, que la tarea que tiene entre manos Heidegger en esta forma delimitativa de presentar su concepción consiste en reconocer cuáles son las bases que justifican la diferencia antes seńalada, por una parte, y a partir de este reconocimiento explicar por qué estas formas de operación conceptual son metódicamente aún limitadas para el ejercicio de la filosofía, de tal manera que resulte necesario desde aquí concebir una tercera posibilidad más originaria, por otra (GA 60, p. 59).

Esta diferencia se justifica, inicialmente, en el esfuerzo de la fenomenología trascendental (Husserl) por conformar para sí la región objetual que le es propia, lo cual depende de la fundamentación de una lógica pura de la objetividad (ontología formal) en la legalidad constitutiva de la conciencia no empírica en cuanto a ella refiere todo posible objeto de conocimiento científico. En otras palabras, la fenomenología trascendental, en esta reconstrucción heideggeriana, está movida por la pretensión de conformar (Ausformen) un campo específico de objetos (la conciencia trascendental) en el cual, sin

${ }^{8}$ Es de notar que el término alemán Allgemeinheit no refiere sólo a la generalidad, sino que cubre también el sentido más amplio en que hablamos en lenguaje técnico filosófico de "universalidad". La generalidad es sólo una forma de universalidad (la del género), pero no toda universalidad es genérica. El problema, como es sabido, es apuntado por Aristóteles en Metafísica III 3 998b22-28 y IX 1 1059b31-34 en el contexto del problema de lo que tradicionalmente se conoce como la universalidad de los trascendentales (ser, uno). Allgemeinheit, por lo pronto, mienta tanto el sentido de universalidad general como de la no general (trascendental). Incluso, dentro del desarrollo histórico-filosófico del concepto, es precisamente esta la palabra que al comienzo de la Fenomenología del Espíritu caracteriza el orden homogéneo e infinitamente divisible del tiempo y del espacio ("Certeza sensible"; HEGEL, 1980, p.68) o la unidad de múltiples propiedades ("Percepción”; HEGEL, 1980, p.72ss).

${ }_{9}$ Heidegger cita en (GA 56/57, p. 57), expresamente Hua (XVIII cap.11) y Hua (III/1, p.31). 
embargo, se da la condición de posibilidad de la constitución (Konstitution) de las relaciones lógicas que determinan a los objetos de conocimiento en general. El núcleo del argumento de Heidegger, siguiendo en esto a Husserl, es que la condición de posibilidad del concepto de "objeto" es completamente libre en relación a la de los conceptos generales, o en otras palabras, que "ser-objeto" es posible de forma independiente del contenido material (del conjunto de notas quiditativas de una cosa, la realidad de la cosa), pues es categoría formal, lo cual implica que es dado sólo a través de una cierta modulación de acto intencional. ${ }^{10}$ En este sentido, esta diferencia implica algo más que una mera distinción entre tipos de conceptos. Ciertamente, el que la distinción entre contenido material/real y categoría formal pueda ser puesta ante la mirada filosófica sólo a través de una reconducción fenomenológica al campo de la intencionalidad, es indicio suficiente de que tal distinción es mucho más compleja que la mera diferencia entre forma y materia. Por consecuencia, abordaré la exposición heideggeriana de esta distinción fenomenológica de forma no enteramente lineal, sino destacando ciertos hitos problemáticos que dan cuenta de una discusión mucho más rica y compleja que como se podría apreciar a primera vista.

Lo primero que uno podría notar es que en estricto rigor la diferencia no es sólo entre formas de conceptos, sino entre relaciones intencionales (Bezüge) en cuya ejecución (Vollzug) estos objetos son dados. La diferencia de formas del concepto se funda en la diferencia modal entre formas de "donación" de los objetos, la cual refiere a formas de relación ejecutiva dentro de una estructura intencionalmente concebida. En este sentido el correlato intencional de lo universal (general) es la generalización, el de forma es la formalización. Ambas son modulaciones de relación que dan tipos de objetos diversos según sea diferente la forma en que dicha relación se ejecute. ${ }^{11}$

Ahora bien, en apariencia, una vía posible para acceder al "objeto en general" es el de la generalización, es decir, "objeto en general" es lo último

\footnotetext{
${ }^{10}$ Con respecto a esto confróntese Hua (XIX/2 p. 657-690). Es extraño que en este contexto Heidegger no refiera expresamente a este importante capítulo. Sí, en cambio, lo hace unos años después en su reconstrucción crítica de los hitos fundamentales de la fenomenología trascendental; cf. (GA 20, p.63 ss).

${ }^{11}$ No me detendré aquí en el difícil problema del paralelismo y diferencia entre la estructura tripartita en que Heidegger describe los elementos básicos de la intencionalidad [Gehaltsinn, Bezugsinn, Vollzugsinn; cf. (GA 60, p. 63); también y especialmente (GA 61, p. 52)] y la bipartita en su versión husserliana (noesis-noema). Remito al lector, no obstante, a la somera pero clara explicación que hace al respecto Rodríguez (1997, p. 51-56).
} 
en un proceso de abstracción generalizadora que parte de determinaciones menos generales. Heidegger lo ilustra mediante un ejemplo: "[...] rojo es un color; el color es una cualidad sensible. O la alegría es un afecto, el afecto es una vivencia. Pareciera incluso que uno puede avanzar más: cualidades en general, vivencias en general son esencias [Wesen]. Rojo, color, cualidad sensible, vivencia, género, esencias, son objetos [Gegenstände]." El sentido de este ejemplo es el siguiente: el proceso de abstracción generalizadora es un progreso que avanza desde estadios (determinaciones) de menor extensión (menos generales) a otros de mayor extensión (más generales). "Ser-objeto" es precisamente determinación que puede ser predicada de todo objeto. De esta forma parece natural pensar que "ser-objeto" es la más extensa de las determinaciones que se puede predicar de cualquier objeto y, por tanto, la más general de todas.

Sin embargo, el punto en el que repara el autor es en el siguiente: el tránsito que va desde "rojo" a "color" y de "color" a "cualidad sensible en general" o de "alegría" a "afecto" y de "afecto" a "vivencia en general" no es del mismo tipo que el que va desde estas determinaciones generales a "objeto". ${ }^{12}$ De esta discontinuidad en la progresión generalizadora (continua desde los predicados más específicos hasta los más generales, discontinua desde estos a "ser-objeto") nos da una cierta justificación en otro lugar:

Tenemos que recorrer todos los grados a partir de la percepción de marrón para poder juzgar... [...] es algo"? ¿No ocurre más bien que en cada uno de los estadios de realidad referidos a las propiedades reales que a ese estadio pertenecen se puede formar el juicio: ... es algo? ¿Y no ocurre que esta última caracterización teorética del simple algo sin más ["ser objeto"] se sale por completo de la sucesión de estadios, de manera que cada estadio la puede motivar? En efecto, en esencia este es el caso—o para ser más exactos... se puede llevar a evidencia que en cada estadio según su sentido se da la posibilidad de mentarlo teóricamente como un simple algo. (GA 56/57, p. 113).

Dejando aparte que aquí denomina "algo" (Etwas) a lo que en el ejemplo anterior llama "objeto", la justificación del punto anterior consiste en que "ser-algo"/"ser-objeto" aplica a cualquiera de los niveles de forma independiente, es decir, su aplicación no incluye a ninguno de los niveles superiores de generalización (mediatos o inmediatos) que sean relativos a

${ }^{12}$ Casi el mismo ejemplo y secuencia en Hua (III/1 p.31) 
ese nivel de orden inferior. Esto es precisamente lo que no sucede con los conceptos generales: por cierto se puede predicar de "rojo" el "ser-cualidad sensible", lo cual implica que, para que verdaderamente aplique, lo considero al menos implícitamente como un color y no, por ejemplo, como un sonido. El "rojo" es "cualidad sensible" en cuanto color. Al contrario, "rojo" es algo, es un objeto, independientemente de su pertenencia a la especie "color" o a su género "cualidad sensible"; "rojo" no es algo, no es objeto en cuanto es "color" o "cualidad sensible", sino directamente, sin más. "Ser-algo"/ "ser-objeto" aplica a cada uno de los niveles en el orden de la generalización, independientemente de la posición relativa de ese nivel en relación a los otros. Es justamente esta peculiaridad de "ser-algo" /"ser-objeto" lo que le permite a Heidegger decir que se "sale" (herausfallen) de la serie progresiva en el orden de la generalización. Si este es el caso, entonces "ser-algo" /"ser-objeto" no son conceptos generales, no son géneros. Por otro lado, la generalidad de lo general caracterizada así, constituye sólo una cierta forma de universalidad.

De esta manera la condición de posibilidad de estos conceptos es distinta de lo general. El pleno contraste que arroja esta diferencia aparece todavía con mayor nitidez cuando Heidegger caracteriza la condición de posibilidad fenomenológica de la primera de las formas del concepto. Así pues se puede preguntar: ¿¿de qué depende la generalización para hacer accesible lo general en sus diversos grados? Esto es lo decisivo desde el punto de vista fenomenológico, pues si consideramos, como más arriba lo he señalado, que los diversos estadios en el orden de menor a mayor universalidad son "dados" a partir de la relación a objetos de tipo generalizadora, la plena ejecución de dicha relación depende de que tal acto "quede atado" (gebunden) a una determinada región de cosas (Sachgebiet/Sachregion): "La secuencia de estadios de las universalidades (géneros y especies) está determinada desde el punto de vista de los contenidos quiditatitivo de las cosas [sachhaltig]).” (GA 60, p.58). Heidegger desarrolla esto un poco más adelante:

La generalización puede ser caracterizada como modo del ordenar [Weise des Ordnens]. Ella efectúa aquí una subordinación de determinadas singularizaciones individuales a un contexto cósico [Sachzusammenhang] más abarcador. Este mismo contexto está a su vez en la posibilidad de ser subordinado a un contexto más general y abarcador. Por esta razón la generalización se ejecuta siempre en una esfera de contenido quiditativo [sachhaltig Sphäre]. Su dirección está establecida por el recto punto de partida del contenido quiditativo [Sachhaltige]. De esta manera la generalización sólo tiene sentido en una actitud teorética [Einstellung], pues el contexto de cosas tiene que estar libre, tiene que haber libre acceso para ordenarlo 
conforme a su completa quididad [Sachhaltigkeit]. La generalización es una determinada ordenación de niveles, y por cierto una ordenación de niveles inmanentes a la cosa, ordenación de determinaciones que se relacionan recíprocamente entre sí de tal forma que la más general apunta a la última. Las determinaciones que generalizan consisten siempre en determinar un objeto desde el punto de vista de su quididad a partir de un [contexto] distinto, de tal modo que lo que determina es introducido como perteneciendo a la región de cosas en la que está el "qué" [Was] que hay que determinar. La generalización es por tanto ordenación, un determinar desde un [contexto] distinto, de modo tal que este otro [contexto], en tanto abarcador, pertenece a la misma región de cosas que lo que hay que determinar. Generalización es subordinar a un contexto cósico diferente. (GA 60, p. 60-61, grifo nosso).

Es en función de esta conexión entre generalización y ordenación, o mejor, de esta explicación de la generalización como la relación ejecutiva propia del ordenar, lo que le permitirá a Heidegger hablar en otro lugar de "conceptos de orden". ${ }^{13}$ De esta manera, la generalización ordenadora se refiere a conceptos que, siguiendo este aspecto que destaca el filósofo, podemos llamar taxonómicos. El punto al que, por tanto, indica Heidegger mediante su actitud escéptica inicial es que el acceso fenomenológico a problemas tales como, por ejemplo, la historia, no se abre por la vía de conceptos taxonómicos o, como él llama a esto, "tipológicamente". ${ }^{14}$ Visto por su reverso, la comprensión y explicitación fenomenológica de los fenómenos no es una ordenación.

Para comprender cabalmente esta conclusión parcial es necesario, sin embargo, poner en contraste la otra forma de operación conceptual: la formalización. No obstante, antes de entrar en este punto, creo necesario dejar apuntados algunos aspectos problemáticos de la presentación heideggeriana de la génesis fenomenológica de los conceptos taxonómicos. Esto supone, por cierto, tener a la vista algunos aspectos de la que la refinadísima teoría husserliana a la que hace referencia esta caracterización heideggeriana de la "generalización", aspectos en los que sin embargo no podré ahondar en consideración a la extensión del presente trabajo. ${ }^{15}$

\footnotetext{
${ }^{13}$ En una anticipación a lo que será expresamente acuñado como "indicación formal”, Heidegger diferencia entre conceptos de orden y conceptos de expresión. Los primeros ordenan objetos, los segundos, en cambio, expresan sentido. (GA 58, p. 143).

${ }^{14}$ Contra el método tipologizador, cf. (GA 58, p. 193).

${ }^{15}$ Algunos trabajos importantes en este sentido: (VIGO, 2001, p. 125-150; LOHMAR, 2004, p. $33-$ 64; DASTUR, 1991, p.37-51; GANDER, 2010; ZAHAVI, 2003, p.7-43).
} 
En primer lugar, es por sí mismo un problema la aparente continuidad en los diversos estadios de la generalización, incluso si se excluye el "ser-objeto". Esto aparece más claro si nos remitimos a otro ejemplo que trae Heidegger en otra de sus lecciones. Así en (GA 56/57, p. 113): “[1] La cátedra es marrón; [2] marrón es un color; el color es un dato sensorial genuino; [3] el dato sensorial es el resultado de procesos físicos o fisiológicos [...]" Nuevamente este ejemplo está al servicio de mostrar la errada tendencia de ubicar el predicado de "objeto"/ "algo" como la culminación del proceso abstractivo generalizador. Las razones son idénticas tanto aquí como en GA 60. Sin embargo, a diferencia del ejemplo anterior aquí aparecen proposiciones que claramente se salen de meras relaciones de subordinación de una especie a un género (con claridad la que he enumerado como [3], que más bien da a entender una relación causal). Por su parte, también [1] y [2] difieren no sólo en grado, sino cualitativamente. Es algo muy diferente lo mentado en la primera proposición (destacar la cualidad sensible de un individuo) que propiamente la subordinación de "marrón" a su género de "color". Lo primero es más bien expresión de la intuición de una cosa reducida a datos sensoriales; lo segundo en cambio supone un nuevo acto de captación-la captación de universales-acto que Husserl llama intuición de esencias o ideación. ${ }^{16}$ Lo importante de retener en este punto es que el proceso de abstracción generalizadora no es tan simple como aparece descrito a primera vista; implica en sí mismo un complejísimo desarrollo de estratos que, desde el punto de vista fenomenológico, difieren ellos también cualitativamente (intencionalmente).

Conectado con lo anterior, en segundo lugar, es de notar la forma en que se articulan estas relaciones taxonómicas, a saber, en la forma de proposiciones (juicios). Este no es un mero detalle, sino que revela, a mi modo de ver, que lejos de haber una mera diferenciación entre generalización y formalización, hay más bien una estructura en que de cierta forma la primera supone la segunda. Si recordamos que el juzgar supone el acto del simple percibir que lo funda, pero que en cuanto mentar demostrativo (hinweisende Meinen) de lo percibido, precisamente lo rebasa de intenciones significativas (momentos sincategoremáticos), ya la proposición del primer nivel— "la mesa es marrón" - implica en orden a destacar una cualidad de un sujeto (en el ejemplo el ser-marrón de la mesa) al menos de forma no temática

${ }^{16}$ Así por ejemplo, siguiendo casi el mismo ejemplo en Hua (II, p. 57); a su vez Hua (XIX/2 p.690691); Hua (III/1 p. 49 ss). También la reconstrucción de Heidegger en (GA 20, p. 90-97). 
tales momentos formales. ${ }^{17}$ Pero en la proposición del segundo nivel—el marrón es un color-, en la medida en que queda supuesta una intuición de universales, presupone, a su vez, como acto de síntesis el objeto percibido fundante, sin mentarlo en la expresión. De hecho, Husserl mismo presenta el acto correlativo a, por ejemplo, "marrón" como el "[...] otro grupo de actos categoriales" (HUA XIX/2, p. 690). Lo que importa retener aquí es que, aun cuando en la caracterización heideggeriana se realce sobre todo la dependencia de la generalización de los contenidos quiditativos — que la generalización se atiene al "qué" y no al "cómo"-, no es esto lo único puesto en juego en la argumentación. Más bien, el que Heidegger introduzca en la caracterización de la generalización la noción fenomenológica de "modo del ordenar", estaría indicando que esta operación conceptual implica otras operaciones más fundamentales que hacen posible esto. ${ }^{18}$

Por último, y en estrecha conexión con lo anterior, si ponemos aparte lo problemático de las distinciones que establece Heidegger entre "región de cosas" (Sachregion), "contexto cósico" (Sachzusammenhang), "contenido quiditativo" (Sachhaltigen), "quididad” (Sachhaligkeit) y "qué" (Was), hay aquí un problema que es necesario destacar. Por lo pronto, no parece claro cuál sea la base para la delimitación de las regiones de cosas. Esto es problemático porque según el sentido del pasaje destacado más arriba no se trata tanto de un mera progresión a grados más generales, sino de la subordinación de individuos a contextos más amplios, lo cual supone que estos contextos están al menos prefigurados de algún modo con anterioridad a dicha subordinación. En dos ocasiones el pasaje parece sugerir esto: "[...] ordenación de determinaciones que se relacionan reciprocamente entre si de tal forma que la más general apunta a la última", lo cual implica que no sólo se progresa hacia los géneros más abarcadores, sino que también estos apuntan en la dirección contraria. En otro pasaje indica la misma idea: "[...] de tal modo que lo que determina es introducido como perteneciendo a la región de cosas en la que está el "qué" [Was]

\footnotetext{
${ }^{17}$ Con respecto a esto Hua (XIX/2 p. 555, p. 667 ss). También la reconstrucción heideggeriana en (GA 20, p. 63-97). Asimismo Vigo (2002, p.187-211).

${ }^{18}$ Lo que sostengo, pero que aquí no podré desarrollar mucho más allá, es que la generalización supone la intuición de esencias, por lo pronto materiales. Esto permite entrever que la crítica de Heidegger apunta, entre otras cosas, a la ideación de esencias formales como método de acceso a los fenómenos por encerrar el peligro de una generalización. Esto lo afirma expresamente el filósofo "La 'esencia' [...] se piensa en cuanto estructurada en esencias genéricas, esencias específicas, esencias individuales. Esto lleva el peligro de equiparar la intuición fenomenológica con la captación de relaciones de orden." (GA 58 , p. 237, traducción de F. de Lara). En el fondo, Heidegger ve que la generalización rebasa el ámbito de las determinaciones reales, pues en el fondo su motivación es la de la ordenación.
} 
que hay que determinar." Así pues, si uno asume que determinar es "subordinar una singularización individual a un contexto más amplio", lo más general aquí se "introduce" en la región de cosas (¿?) a la que pertenece el individuo que hay que determinar desde el punto de vista de su quididad. Luego, para que este acto de ordenación de cosas mediante subordinaciones funcione, tiene que ceñirse a una región predelinieada de algún modo. Precisamente la ordenación por subordinación se da en una región de cosas ya prefigurada con anterioridad a dicha ordenación. Esto apunta de forma tácita a uno de los núcleos de la pretensión científica de la fenomenología husserliana, que el autor presenta al inicio mismo de sus Ideas, a saber, la dependencia de las ciencias de hechos de las ontologías regionales (ciencias de esencias), las cuales a su vez dependen de la "ciencia del objeto en cuanto tal", es decir, de la "ciencia de los conceptos puramente lógicos” (HUA III/1, p. 23). ${ }^{19}$

El resultado de esta discusión crítica de la generalización y de sus conceptos taxonómicos es, por una parte, relativamente clara, a saber, que en verdad se dan conceptos, que escapan ellos mismos a toda generalización y que no se constituyen como conceptos taxonómicos a partir de una ordenación. Los conceptos de "objeto", "algo" (entre otros) ${ }^{20}$ son correlato de otra operación conceptual. Pero, por otra parte, si nos atenemos a la compleja doctrina de base a la que esta reconstrucción hace referencia se dan también otros resultados tal vez menos evidentes, pero igualmente decisivos. Esto último indica en la siguiente dirección: no sólo conceptos tales como "objetos" no son taxonómicos, es decir, salen del marco de una ordenación, sino que son su presupuesto. En otras palabras, a lo que implícitamente estaría apuntando Heidegger es a que la generalización debe su posibilidad de ejecución a una relación intencional básica que refiere desde la partida a objetos con anterioridad a toda ordenación de los mismos. Esta relación básica supuesta por la generalización es la Einstellung (actitud teórica). Es lo que Heidegger señala a la pasada, pero de forma bien elocuente: "De esta manera la generalización sólo tiene sentido en una actitud teorética, pues el contexto de cosas tiene que estar libre, tiene que haber libre acceso para ordenarlo conforme a su completa quididad”. (GA 60, p. 61, grifo nosso).

\footnotetext{
19 Este problema teórico sistemático propio de la fenomenología entendida como lógica del conocimiento científico, es radicalmente transformado en Heidegger. Lo que ya en estas lecciones tempranas se echa de ver con respecto a la condición de posibilidad fenomenológica de las ciencias no filosóficas, es que debe ser explicitada su génesis desde un marco metódico completamente diferente (génesis existencial de las ciencias). (GA 58, p. 65-90).

${ }^{20}$ La gama de categorías formales es ciertamente más amplia. Algunos ejemplos en Hua (III/1 p.28).
} 
Ahora bien, si la generalización sólo tiene sentido en la relación intencional básica en modo teorético, y este de suyo refiere a objetos, y si la condición misma de objeto es lo que hace expreso la formalización, entonces en cierto sentido no se trata de una mera distinción entre diversas operaciones conceptuales, sino que a su vez de una jerarquización entre operaciones más o menos fundamentales.

\section{DisCuSión CRÍTICA DE LA "FORMALIZACIÓN"}

El aspecto decisivo de la frase recién citada es que caracteriza la generalización precisamente como aquello que permite establecer la conexión de dependencia con la formalización. En efecto, aquí la generalización aparece como actitud teorética (Einstellung). Ahora bien, ¿en qué medida esta caracterización permite establecer esta relación de dependencia de la generalización? ¿Cómo caracteriza Heidegger en concreto la formalización? El punto de partida precisamente es el de la condición que hace posible el predicado de "objeto" ("El rojo es objeto", "el color es objeto", "la propiedad sensible es objeto"), lo que motiva dicha predicación. En este sentido, la caracterización de la formalización es el reverso de la generalización, pues si efectivamente-como se ha mostrado-ser-objeto es un predicado tal que surge con independencia de los contenidos quiditativos que hacen posible los conceptos taxonómicos de la generalización, entonces, desde un punto de vista estrictamente fenomenológico, la génesis del carácter de objeto de todo objeto reside en el “[...] sentido de la actitud teórica como tal." (GA 60, p.61). Dicho de otra forma, el origen del carácter objetivo de todo objeto de conocimiento emerge expresamente sólo cuando se toma en consideración el modo de relación (Bezug) que refiere a aquel como su correlato intencional. $\mathrm{Y}$ es este paso el que opera la formalización. De esta manera, la formalización hace explícito el carácter meramente formal del objeto en cuanto tal en la medida en que reconduce la consideración hacia la relación que refiere a objetos precisamente en el modo en que tiene sentido referir a ellos como tales. Tiene sentido referir a objetos precisamente en la actitud teórica, independientemente del contenido que estos objetos presenten para su ordenamiento taxonómico. Esta correlación entre actitud teórica y objeto que hace expresa la formalización la explica Heidegger de la siguiente manera:

La formalización no está atada a un determinado qué de un objeto determinado. La determinación evade el contenido quiditativo del objeto y considera el objeto por el respecto de que él está dado; el objeto 
es determinado como captado, como aquello "a lo cual" apunta la relación cognitiva. Esta relación teórica sólo dice del "objeto en general" que es el "a lo cual” de la relación de la actitud teórica. (GA 60, p. 59).

Tres aspectos son importantes de destacar en esta somera reconstrucción que hace Heidegger de uno de los aspectos centrales de la fenomenología husserliana. En primer lugar, aun cuando el acto de hacer expreso el carácter de objeto de todo objeto de conocimiento mediante la formalización no sea condición para la ordenación taxonómica de una región de objetos, el carácter de objeto y su correlativo intencional, a saber, la relación de actitud teórica, si son condición lógica y ontológica de toda generalización. De ahí que está última sea caratulada de plano por el filósofo como actitud teórica. Toda ordenación taxonómica de contenidos quiditativos supone formalmente lo que la formalización hace expreso: ser-objeto. Es en este sentido que la generalización es "posterior" a la formalización, o dicho con más precisión, es dependiente en el orden lógico y ontológico de fundación de lo que la formalización hace expreso mediante su reducción. El carácter formal de objeto es condición lógica y ontológica de posibilidad de toda ordenación taxonómica de contenidos quiditativos, en la medida en que esta ordenación supone el haberse instalado desde ya en la actitud teórica: la del puro captar orientado directamente hacia el objeto que sólo en cuanto tal es capaz de exhibir contenidos quiditativos.

En segundo lugar, lo que se hace expreso mediante la formalización, a pesar de ser indeterminado desde el punto de vista de sus contenidos, no es algo simple. Al contrario, implica una trama compleja, que es precisamente en la que pretende hacer luz la fenomenología. Si es verdad que el todo de categorías formales depende en último término del sentido de relación de la actitud teórica, entonces esta última implica una multiplicidad organizada u organizable al menos. ${ }^{21}$ La multiplicidad compleja de lo categorial formal implica, por eso mismo, una complejidad en la correlación intencional. Por ello, finalmente- $-\mathrm{y}$ este es el punto en el que Heidegger presiona aquí de forma crítica - para la fenomenología formal, esta correlación intencional que supone el carácter categorial y formal de todo objeto de conocimiento, puede ser precisamente referida a su vez como un nuevo campo de objetos de conocimiento a través de la formalización. Es decir, la multiplicidad de lo

${ }^{21}$ Expresamente expuesto de esta forma por Husserl en su escrito programático Die Idee der Phenomenologie, cfr. (HUA II, p.57). 
categorial en su correlación intencional puede ser conformada como una "región formal" para una ciencia fenomenológica que apunta a ella a su vez como objeto.

Ahora bien, si esto es así, entonces lo formal—el todo categorial de lo objetivo reconducido a sus correlaciones intencionales, es decir, visto en correspondencia con sentido de relación que a ello refiere y ejecuta—no ofrece directamente la base para una ordenación taxonómica, pero como "región conformada" puede ser puesto como una multiplicidad que indirectamente puede ser ordenada teóricamente. El punto crítico para Heidegger es precisamente este, a saber, que "La formalización es primariamente ordenación sólo a través de la conformación [de una región]." (G.A 60, p.59). Es decir, lo paradójico en el núcleo mismo de la fenomenología formal consiste en que: 1) el objeto es formalmente (sin consideración de sus contenidos) reconducido a su correlación intencional en el sentido de relación de la actitud teórica; 2) que la conformación de la "región formal-ontológica" como campo de conocimiento científico supone a su vez la conformación de la multiplicidad del sentido de relación de la actitud teórica como región que puede ser ordenada mediante conceptos de generalización. ${ }^{22} \mathrm{El}$ segundo punto es el problemático, pues lo que parece estar sugiriendo Heidegger es que para explicitar fenomenológicamente el ámbito originario de lo formal (la intencionalidad) se cae nuevamente en una forma de conceptualización que es precisamente derivada de este origen; y aún más, si es verdad que la actitud teórica es un modo en que se articula el sentido de relación y de ejecución, no puede ella misma, en cuanto modo del sentido de relación ejecutiva, ser abordada desde este mismo modo. En otras palabras, la crítica de Heidegger no se dirige a la formalización por sí misma, sino a aquella motivación a la que sirve, a saber, el intento de volver teóricamente-lo cual incluye la estructura conceptual de tipo taxonómico- a la unidad en sí misma compleja de la actitud teórica, entendida esta como condición fundamental del todo

\footnotetext{
${ }^{22}$ Es más que posible que en su crítica Heidegger esté pensado en textos de Husserl como el siguiente: "Uno debe distinguir múltiples formas y especies del conocimiento y no solo esto: cabe investigar, además, los modos como estas formas y especies se relacionan entre sí. Pues entender el conocimiento significa aclarar genéricamente los nexos teleológicos del conocimiento, que terminan en ciertas relaciones esenciales entre diversos tipos esenciales de formas intelectuales. Y esto incluye también a la aclaración última de los principios que, como condiciones ideales de la posibilidad de la objetividad científica, sirven como normas que regulan todo el procedimiento de la ciencia empírica. Toda la investigación encaminada a la aclaración de los principios se mueve por entero en la esfera de las esencias, la cual, a su vez, se constituye sobre el fondo de fenómenos singulares de la reducción fenomenológica." (HUA II, p.57-58, traducción de J.A Escudero, grifo nosso).
} 
intencional en el que se dan propiamente objetos. Parece al menos implicar esto una cierta violación de principio, pues si lo originario de la actitud teórica con respecto a la constitución del objeto como tal estriba en que es un modo de ser relación ejecutiva, al ser abordada desde esta misma actitud teórica se pierde precisamente esta carácter originario: el de ser relación ejecutiva. De esta manera, desde el punto de vista de Heidegger, la formalización es insuficiente al menos en uno de los aspectos implicados en su operación, a saber, el de cumplir adecuadamente la referencia al origen (el propio carácter ejecutivorelacional) desde el que surge expresamente el objeto en sentido formal.

Es cierto que Heidegger en su insistente controversia con Husserl tiene a la vista también otros aspectos. Así pues, es evidente que en otros lugares el centro de la crítica reside en la desfiguración de la vivencia de la cosa del mundo que opera inadvertidamente la actitud teórica y la teoría del conocimiento. En efecto, el así llamado "primado de lo teórico" en la filosofía desfigura desde la partida la vivencia de la cosa al describirla en términos de mera percepción de datos sensoriales. ${ }^{23} \mathrm{~A}$ su vez, también es de peso el que, como el texto de GA 60 parece sugerirlo, ${ }^{24}$ el objeto formalmente intuido constituye al fin y al cabo una nueva forma de universalidad no taxonómica, que empero es condición de posibilidad necesaria para toda taxonomía. Sin embrago, a mi entender, esto no constituye al menos en esta primera exposición del problema, el centro de la argumentación. Con respecto a la pregunta de si acaso con la formalización se ha ganado el pleno acceso a los fenómenos, la respuesta en este caso sería negativa, no tanto por la tendencia inmanente de la formalización a lo libre del contenido quiditativo, sino porque el contexto metódico al que pertenece, supone - a partir de sus propias pretensiones de cientificidad acríticamente asumida - la sumisión a principios de ordenación generalizadora, los cuales, no obstante, en cuanto formas de conceptualización son en el fondo derivados. Por eso es que uno puede ver cómo en la discusión anterior la caracterización de la generalización se avanza desde el rasgo que enfatiza su dependencia de los contenidos reales hacia lo que en el fondo le interesa a Heidegger para

\footnotetext{
${ }^{23}$ Es la dirección fundamental que toma en Heidegger (GA 56/57, p. 73-78, 96-101).

${ }^{24}$ Mediante la peculiar ambigüedad del término "allgemein", que como lo dijimos antes puede significar tanto universalidad (no genérica) como generalidad, Heidegger se permite decir: "Das Gemeinsame von Formalisierung un Generalisierung ist, da $\beta$ sie dem Sinn von 'allgemein' stehen, wärend die formale Anzeige mit Allgemeinheit nichts zu tun hat". Valen aquí las mismas indicaciones hechas en nota. 6, con el agravante aquí de que Heidegger se distancia con la indicación formal de toda forma de universalidad. Acerca del radical rechazo de toda forma de universalidad del concepto filosófico (GA 58, p.187). Esto entrańa, como algunos autores lo han hecho ver, un peculiar problema en el nivel de la acreditación y “desformalización” (ejecución) de lo formalmente indicado. (GETHMANN 1993, p. 270).
} 
incluir en ello a la fenomenología husserliana: la generalización es un modo del ordenar.

Heidegger es en otro lugar completamente prístino en este punto:

Filosofía trascendental — no es inadecuada por provenir hasta ahora del primado del conocimiento teórico y por permitir que todos los problemas filosóficos estén dominados por él, sino porque en general toda ciencia de la conciencia.....tiende a una analítica sistemática y a una deducción, cuya posibilidad ideal tiene ya como motivo el ideal de conocimiento anteriormente mencionado [conocimiento sometido al método ordenador] (que estos objetos estén en una dimensión pretendidamente distinta de formas categoriales no cambia por principio su postura de fondo). (GA 58, p. 144, traducción F. de Lara).

De esta manera la crítica de Heidegger no se dirige directamente contra la formalización, sino contra la limitación a que queda sujeta en la medida en que la fenomenología husserliana queda en conjunto metódicamente inmune a la tendencia originaria que despunta en la formalización.

\section{INDICACIÓN FORMAL COMO PRINCIPIO METÓDICO DE LA INVESTIGACIÓN}

Si se acepta que lo argumentado más arriba constituye uno de los núcleos de la crítica heideggeriana a la fenomenología precedente, se acepta entonces que la tendencia de la fenomenología hermenéutica a la formalidad no es abandonada por Heidegger, sino que acentuada, o mejor, llevada a un plano aún más radical. Para decirlo de otro modo, la radicalización fenomenológica que intenta Heidegger a través de la indicación formal se la puede entender como una acentuación de la tendencia a lo formal de la propia fenomenología. De hecho, la pretensión de este filósofo es que la indicación formal es "formal" en un sentido "más originario" (GA 60, p. 59) que el de la formalización. Pues si es ya un paso decisivo comprender que lo formal en la formalización teóricamente motivada se cumple con independencia de los contenidos quiditativos, es decir, si lo decisivo estriba en concebir que es condición de posibilidad fenomenológica para la explicitación de las categorías formales su reconducción al sentido de una relación-ejecutiva (la actitud teorética), resulta, sin embargo, un prejuicio infundado cuando se asume acríticamente que el acceso fenomenológico mismo a este todo intencional (actitud teóricaobjeto como tal) se articula a su vez desde principios de ordenación propios de 
la actitud teorética. Lo formal concierne también a este segundo nivel, es decir, no sólo se opera una desconexión con respecto a los contenidos quiditativos en el orden del objeto, sino que también "se deja en vilo" (GA 60, p.63) el sentido de relación ejecutiva en el plano del acceso fenomenológico a la estructura intencional íntegra: "La relación y ejecución del fenómeno no es determinada de ante mano, sino que se la deja en vilo." (GA 60, p. 63). La indicación formal refiere eminentemente a lo que en la estructura intencional es "según la forma de relación” (GA 60, p.63). Eso a lo que refiere la indicación formal es mentado de tal manera — en su carácter ejecutivo relacional— que la forma de acceso a ello queda necesariamente "libre", es decir, no prejuzgada en el modo de su relación ejecutiva; queda, en este sentido, indeterminada.

Esta es, a mi entender, la razón principal por la cual Heidegger, ya a esta altura, considera que la filosofía no es en el fondo ninguna ciencia teórica ni se constituye esencialmente como una teoría (GA 60, p. 62), sino que es ciencia originaria (Urwissenschaft). ${ }^{25}$

Esta caracterización de la indicación formal se constituye, por una parte, a partir de un contraste principalmente con la formalización, pero por otra, esta delimitación frente a la formalización está dominada por la pretensión de radicalizar la tendencia hacia lo formal inherente a la fenomenología. Ahora bien, dado este enfoque, hay dos aspectos de la indicación formal que es necesario destacar con mayor énfasis. Primero, lo que algunos autores han llamado su "función disuasiva" (die abwehrende Funktion des formales Anzeige; DE LARA, 2008, p. 187). Esto quiere decir que lo que hace la indicación formal es advertir negativamente-dice: ¡no! (GA 60, p. 63) —, y esto al menos en dos direcciones. Por una parte, en la medida en que aparta desde el inicio la posibilidad de considerar eso a lo que refiere la indicación formal (lo que en este contexto llama el fenómeno) como un objeto (Objekt). Eso con lo que trata la fenomenología, mediante la indicación formal, no tiene la forma de lo objetual (Objetktmässige). Pero justo por esto la indicación formal indica negativamente en la dirección complementaria, a saber, cómo no hay

\footnotetext{
${ }^{25}$ Esta forma de determinar la filosofía cobra especial fuerza en GA 58. Precisamente es esta lección la que se dedica íntegramente a concretar la indicación formal contenida en esta expresión. Cabe resaltar el doble sentido de lo originario: por un lado en cuanto refiere al ámbito originario; pero todavía de forma más acentuada en cuanto refiere a ello (adverbialmente) de forma originaria y con ello se toma a sí misma originariamente entre manos. Esta caracterización adverbial de lo originario de esta ciencia la recoge Heidegger ya al inicio "[...] sólo la auténtica y concreta realización y efectuación (observancia) de las "tendencias" que actúan en ella misma [la ciencia originaria] la llevan a ella misma y a su campo de problemas." (GA 58, p. 2, traducido por F. de Lara).
} 
que abordar el fenómeno. Esto implica que la indicación formal, por la vía de la negación, prescribe que la forma de acceso fenomenológico al fenómeno no está asegurada desde la partida. A través de esta disuasión la indicación formal deja libre (aún no determinada) la posibilidad del acceso fenomenológico al fenómeno en cuanto tal.

Esta última de las consecuencias de la caracterización de la indicación formal en términos negativos es crucial para entender su lugar metódico, nuevamente por vía negativa. No podré aquí, sin embargo, extenderme en detalle.

El punto consiste en que justamente este carácter negativo de la indicación formal es indicio a su vez de que casi pareciera que es en todo respecto imposible comenzar siquiera con algún ejercicio de conceptualización. En efecto, si precisamente la indicación formal neutraliza las bases mismas de cualquiera aproximación teórica, a saber la consideración de que se trata con objetos que pueden ser ordenados mediante conceptos de corte taxonómico; si la indicación formal pone el énfasis en la peculiar indocilidad categorial de los fenómenos a ser considerados desde la partida como objetos y ser tratados teóricamente en consecuencia- es decir, dentro del marco de conceptos de orden-, entonces no es raro que surja necesariamente el desconcierto frente al mismísimo punto de partida de un ejercicio fenomenológico. ¿Con qué se parte? ¿Cómo se parte? Esto, a mi entender, no es casual sino que constituye el núcleo de la indicación formal, a saber, que la pretensión de Heidegger está en extremar a tal punto las cosas que de algo tal como la indicación formal depende el punto de partida, el "principio", de la investigación fenomenológica, por lo pronto como para parecer poder impedirlo. Dicho de otra forma, lo que se pretende recoger mediante la indicación formal no es meramente una forma del concepto distinta a la habitual, sino la de aquellos conceptos que sirven de punto de partida para la filosofía. Toda esta caracterización por vía negativa de la indicación formal apunta en el fondo al problema del punto de partida de la filosofía. Ella tiene, recordémoslo, "el significado de punto de partida de la explicación fenomenológica”. Esto incide de manera decisiva incluso en la modulación misma que toma el principio visto desde su carácter de principio. Pues es justamente a partir de esta modulación en la concepción misma de los conceptos filosóficos que el principio en filosofía no puede significar ya lo más general de todo, lo que es válido para todos los casos, sino que se transforma metódicamente en aquella indicación que abre una dirección para la ejecución de un posible itinerario de investigación en la medida en 
que es el cumplimiento mismo de ese itinerario el que concreta y acredita el principio asumido. ${ }^{26} \mathrm{Y}$ esto implica que la indicación formal en cuanto principio metódico pone a la propia investigación filosófica en guardia contra su propia tendencia a asumir sin crítica los presupuestos desde los que parte, como el hecho mismo de caracterizarse a sí misma como una ciencia y desde la actitud teórica. Es en este sentido que a través de la indicación formal y como indicación formal el principio no es sólo principio, sino que es principio en cuanto es tomado metódicamente de forma "principial" (prinzipiell).

ABALO, Francisco C. The formal indication as start point of the phenomenological investigation. Tans/form/açâo, Marília, v. 40, n. 4, p. 67-88, Out./Dez., 2017.

\begin{abstract}
The aim of this article is to focus on central aspects of Heidegger's conception of the formal indication. An early Heideggerian exposition of this meta-concept take as starting point a differentiation from others conceptual operations (generalization and formalization). First, it will be critical discuss the exposition of the generalization. The main point is that the distinction of generalization and formalization is rather a foundational structure than a simple difference. This premise leads to the view that the Heidegger's critical attempt is not addressed to the formalization as such, but rather to the methodological context (transcendental phenomenology) to which the formalization serves. Finally, based on this, it will be claimed that is a necessary condition for the determination of the formal indication as a methodological principle of the phenomenological investigation, that this meta-concept is a radical tendency toward the formal.
\end{abstract}

KEYWORDS: Heidegger. Husserl. Phenomenology. Hermeneutic. Formal indication.

\title{
REFERENCIAS
}

ARISTÓTELES: Aristotle's metaphysics. Revisión del texto, introd. y comentario de W. D. Ross. Oxford: Clarendon, 1924.

DASTUR, F. Heidegger und die logische untersuchungen. Heidegger-Studien, v. 7, p. 37-57, 1991.

DE LARA, F. Phänomenologie der möglichkeit: grundzüge der philosophie Heideggers 1919-1923. Freiburg; München: Karl Alber, 2008.

GANDER, H.-H. (Ed.). Husserl lexicon. Darmstadt: Wissenschaftliche Buchgesselschaft, 2010 .

${ }^{26}$ Toda esta nueva dimensión de la indicación formal, en la que no puedo entrar en detalle aquí, es expresamente tratada en (GA 61, p. 16-39). Ahí el acento está puesto en el carácter de principio de la indicación precisamente en cuanto indica hacia algo y no sólo disuade de algo, es decir, en cuanto su sentido supone una "desformalización" en el plano ejecutivo existencial. Sigo en estas escuetas líneas la excelente interpretación que en este punto hace Rodríguez (1997, p.162 ss). 
GETHMANN, C-F. Philosophie als vollzug und al bezug. In: Dasein: erkennen und handeln. Heidegger im phänomenologischen kontext. Berlin; New York: Walter de Gruyter, 1993. p. 248-280.

HEGEL, G. F. W. Phänomenologie des geistes. Hrsg. von H. F. Wessels \& H. Clairmont, Hamburg: Felix Meiner, 2006.

HEIDEGGER, M. Prolegomena zur geschichte der zeitbegriff. In: Gesamtausgabe Band 20 Freiburg am Main: Vittorio Klostermann, 1979.

Phänomenologische interpretation zu Aristoteles: Einfúhrung in die phänomenologische forschung. In: Gesamtausgabe Band 61. Freiburg am Main: Vittorio Klostermann, 1985.

. Gesamtausgabe Band 56/57: zur bestimmung der philosophie. Freiburg am Main: Vittorio Klostermann, 1987.

. Sein und zeit. Tübingen: Niemeyer, 1993.

. Gesamtausgabe Band 58: grundprobleme der phänomenologie (1919/1920). Freiburg am Main: Vittorio Klostermann, 1994.

. Gesamtausgabe Band 60: phänomenologie des religiösen Leben. Freiburg am Main: Vittorio Klostermann, 1995.

. Gesamtausgabe Band 9: wegmarken. Freiburg am Main: Vittorio Klostermann, 1996.

Problemas fundamentales de la fenomenología (1919-1920). Traducido por Francisco de Lara. Madrid: Alianza, 2014.

HUSSERL, E. Die idee der phänomenologie: fünf vorlesungen. Haag: Nijhoff, 1950. (Husserliana Band II).

. Idee zu einer reinen phänomenologie und phänomenologische philosophie: Erstes. Buch: allegemeine einfhührung in die reine phänomenologie. Haag: Nijhoff, 1952. (Husserliana Band III/1).

XVIII/XIX).

Logische untersuchungen Bd. I-II/1-2.Haag: Nijhoff, 1975-1984. (Husserliana

- La idea de fenomenología. Traducido por Jesús Adrián Escudero. Barcelona:

Herder, 2011.

LOHMAR, D. El concepto de la intuición categorial en Husserl. Traducido por Kurt Späng. Anuario Filosófico, v. XXXVLL, n. 1, p. 33-64, 2004.

RODRÍGUEZ, R. La transformación hermenéutica de la fenomenología. Madrid: Tecnos, 1997.

La exposición de la tarea de un análisis preparatorio del Dasein. In:

(Coord.). Ser y tiempo de Martín Heidegger: un comentario fenomenológico. Madrid: Tecnos, 2015. p. 67-78. 
VIGO, A. Constitución, objetividad categorial y modalidad en Husserl: la realización del modelo teórico de las logische untersuchungen en las Vorlesungen über Bedeutungslehre de 1908. Escritos de Filosofía, Buenos Aires, v. 39-40, p. 125-150, 2001. . Intuición categorial. Themata, Sevilla, n. 28, p. 187-212, 2002.

XOLOCOTZI, A. Fenomenología de la vida fáctica: Heidegger y su camino a ser y tiempo. Madrid: Plaza y Valdés, 2004

ZAHAVI, D. Husserl's phenomenology. Stanford: Stanford University Press, 2003.

Recebido em 19/02/2016

Aceito em 26/12/2016 